\title{
Front Matter: Volume 6950
}

, "Front Matter: Volume 6950," Proc. SPIE 6950, Laser Radar Technology and Applications XIII, 695001 (13 May 2008); doi: 10.1117/12.801207

SPIE Event: SPIE Defense and Security Symposium, 2008, Orlando, Florida, SPIE. United States 


\title{
PROCEEDINGS OF SPIE
}

\section{Laser Radar Technology and Applications XIII}

\author{
Monte D. Turner \\ Gary W. Kamerman \\ Editors \\ 19-20 March 2008 \\ Orlando, Florida, USA \\ Sponsored and Published by \\ SPIE
}

Volume 6950 
The papers included in this volume were part of the technical conference cited on the cover and title page. Papers were selected and subject to review by the editors and conference program committee. Some conference presentations may not be available for publication. The papers published in these proceedings reflect the work and thoughts of the authors and are published herein as submitted. The publisher is not responsible for the validity of the information or for any outcomes resulting from reliance thereon.

Please use the following format to cite material from this book:

Author(s), "Title of Paper," in Laser Radar Technology and Applications XIII, edited by Monte D. Turner, Gary W. Kamerman, Proceedings of SPIE Vol. 6950 (SPIE, Bellingham, WA, 2008) Article CID Number.

ISSN 0277-786X

ISBN 9780819471413

Published by

SPIE

P.O. Box 10, Bellingham, Washington 98227-0010 USA

Telephone +1 3606763290 (Pacific Time) · Fax +1 3606471445

SPIE.org

Copyright (C 2008, Society of Photo-Optical Instrumentation Engineers

Copying of material in this book for internal or personal use, or for the internal or personal use of specific clients, beyond the fair use provisions granted by the U.S. Copyright Law is authorized by SPIE subject to payment of copying fees. The Transactional Reporting Service base fee for this volume is $\$ 18.00$ per article (or portion thereof), which should be paid directly to the Copyright Clearance Center (CCC), 222 Rosewood Drive, Danvers, MA 01923. Payment may also be made electronically through CCC Online at copyright.com. Other copying for republication, resale, advertising or promotion, or any form of systematic or multiple reproduction of any material in this book is prohibited except with permission in writing from the publisher. The CCC fee code is $0277-786 \mathrm{X} / 08 / \$ 18.00$.

Printed in the United States of America.

Publication of record for individual papers is online in the SPIE Digital Library.

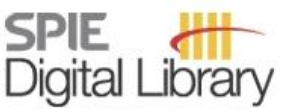

SPIEDigitallibrary.org

Paper Numbering: Proceedings of SPIE follow an e-First publication model, with papers published first online and then in print and on CD-ROM. Papers are published as they are submitted and meet publication criteria. A unique, consistent, permanent citation identifier (CID) number is assigned to each article at the time of the first publication. Utilization of CIDs allows articles to be fully citable as soon they are published online, and connects the same identifier to all online, print, and electronic versions of the publication. SPIE uses a six-digit CID article numbering system in which:

- The first four digits correspond to the SPIE volume number.

- The last two digits indicate publication order within the volume using a Base 36 numbering system employing both numerals and letters. These two-number sets start with 00, 01, 02, 03, 04, 05, $06,07,08,09,0 A, 0 B \ldots 0 Z$, followed by $10-12,20-2 Z$, etc.

The CID number appears on each page of the manuscript. The complete citation is used on the first page, and an abbreviated version on subsequent pages. Numbers in the index correspond to the last two digits of the six-digit CID number. 


\section{Contents}

vii Conference Committee

3D IMAGING LIDAR SYSTEMS

695002 High resolution ladar using time-correlated single-photon counting [6950-01]

O. Steinvall, L. Sjöqvist, M. Henriksson, P. Jonsson, Swedish Defence Research Agency (Sweden)

695004 Three dimensional imaging laser radar system for short-range target detection and identification [6950-03]

P. Li, K. Li, H. Chen, W. Gou, Beijing Institute of Technology (China)

695007 Inflight performance of a second-generation photon-counting 3D imaging lidar [6950-06] J. Degnan, R. Machan, E. Leventhal, D. Lawrence, G. Jodor, C. Field, Sigma Space Corp. (USA)

\section{COHERENT LIDAR APPLICATIONS I}

695008 Ultra-fast coherent optical system for active remote sensing applications [6950-07]

S. Datta, D. Becker, A. Joshi, R. Howard, Discovery Semiconductors, Inc. (USA)

695009 Coherent lidar imaging of dust clouds: waveform comparison with the poly-phase (P4) modulation waveform [6950-08]

D. G. Youmans, SPARTA, Inc. (USA)

6950 0A Resonance Raman measurements utilizing a deep UV source [6950-09]

A. Willitsford, The Pennsylvania State Univ. (USA); C. T. Chadwick, H. Hallen, North Carolina State Univ. (USA); C. R. Philbrick, The Pennsylvania State Univ. (USA)

6950 OB Supercontinuum lidar applications for measurements of atmospheric constituents [6950-10] D. M. Brown, Z. Liu, C. R. Philbrick, The Pennsylvania State Univ. (USA)

\section{LADAR SYSTEM MODELING AND CALIBRATION}

6950 OE Modeling the detection of optical sights using retro-reflection [6950-15]

A. L. Mieremet, R. M. A. Schleijpen, TNO Defence, Security and Safety (Netherlands);

P. N. Pouchelle, ENSIETA (France)

6950 OF Multiscale target manifold characterization for 3D imaging ladar [6950-16]

E. Whittenberger, D. Waagen, N. Shah, D. Hulsey, Raytheon Missile Systems (USA) 
$6950 \mathrm{OH}$ Calibration of full-waveform airborne laser scanning data for object classification [6950-19] C. Briese, B. Höfle, H. Lehner, W. Wagner, Vienna Univ. of Technology (Austria);

M. Pfennigbauer, A. Ullich, RIEGL Laser Measurement Systems GmbH (Austria)

6950 Ol Simulation of a new 3D imaging sensor for identifying difficult military targets [6950-20] C. Harvey, J. Wood, P. Randall, G. Watson, QinetiQ Ltd. (United Kingdom); G. Smith, Selex Sensors and Airborne Systems Ltd. (United Kingdom)

6950 0J ASTM E57 3D imaging systems committee: an update [6950-32]

G. S. Cheok, A. M. Lytle, K. S. Saidi, National Institute of Standards and Technology (USA)

6950 OK Real time processing enables fast 3D imaging at single photon level [6950-33]

I. Bakalski, LIDAR Technologies Inc. (USA); J. Pereira Do Carmo, European Space Agency (Netherlands); S. Bellis, SensL (Ireland); R. Bond, ABSL Space Products (United Kingdom); M. Himphries, Sula Systems Ltd. (United Kingdom); M. Foster, LIDAR Technologies Ltd. (United Kingdom); C. Jackson, SensL (Ireland); D. Reece, ABSL Space Products (United Kingdom); S. Sutton, LIDAR Technologies Ltd. (United Kingdom)

$6950 \mathrm{OL}$ Overview of LMCT's advanced ladar signal simulator (ALASS) [6950-34]

D. Jacob, P. Gatt, T. Nichols, Lockheed Martin Coherent Technologies (USA)

\section{EMERGING TECHNOLOGIES FOR LIDAR}

6950 OM Holographic optical elements for lidar systems [6950-21]

O. Asmolova, P. Molchanov, Aerospace Mass Properties Analysis, Inc. (USA); R. Billmers, M. Ludwig, RL Associates (USA); V. M. Contarino, Aerospace Mass Properties Analysis, Inc. (USA)

6950 ON Single photon counting Geiger mode InGaAs(P)/InP avalanche photodiode arrays for 3D imaging [6950-22]

R. Sudharsanan, P. Yuan, J. Boisvert, P. McDonald, T. Isshiki, S. Mesropian, E. Labios, Boeing Spectrolab, Inc. (USA); M. Salisbury, Boeing SVS, Inc. (USA)

$695000 \quad$ Large-area high-speed InGaAs photodetectors [6950-23]

H. Yuan, J. Kim, G. Apgar, J. Laquindanum, K. Song, J. Kimchi, T. Wong, Teledyne Judson Technologies (USA)

6950 OP Low-cost ladar imagers [6950-24]

S. Vasile, J. Lipson, aPeak, Inc. (USA)

$69500 Q \quad$ Single-photon-sensitive linear-mode APD ladar receiver developments [6950-25]

G. M. Williams, M. A. Compton, A. S. Huntington, Voxtel Inc. (USA)

IMAGING THROUGH OBSCURANTS

6950 OS An approach to target detection in forested scenes [6950-26]

C. Grönwall, T. Chevalier, G. Tolt, Swedish Defence Research Agency (Sweden);

P. Andersson, Saab Bofors Dynamics AB (Sweden) 
6950 ОT Lidar for obstacle detection during helicopter landing [6950-27]

X. Zhu, P. Church, M. Labrie, Neptec Design Group Ltd. (Canada)

6950 OU Three-dimensional laser scanners with echo digitization [6950-28]

M. Pfennigbaver, A. Ullrich, RIEGL Laser Measurement Systems GmbH (Austria)

6950 OV Experimental validation of ship identification with a laser range profiler [6950-29]

J. C. van den Heuvel, TNO Defence, Security and Safety (Netherlands); P. Pace, Defence Research and Development Canada (Canada); H. H. P. Th. Bekman, F. J. M. van Putten,

R. M. A. Schleijpen, TNO Defence, Security and Safety (Netherlands)

6950 OW Search-lidar demonstrator for detection of small sea-surface targets [6950-30]

J. C. van den Heuvel, H. H. P. Th. Bekman, F. J. M. van Putten, L. H. Cohen,

R. M. A. Schleijpen, TNO Defence, Security and Safety (Netherlands)

Author Index 
Downloaded From: https://www.spiedigitallibrary.org/conference-proceedings-of-spie on 26 Apr 2023

Terms of Use: https://www.spiedigitallibrary.org/terms-of-use 


\title{
Conference Committee
}

\author{
Symposium Chair
}

Larry B. Stotts, Defense Advanced Research Projects Agency (USA)

Symposium Cochair

Ray O. Johnson, Lockheed Martin Corporation (USA)

Program Track Chair

Gary W. Kamerman, FastMetrix, Inc. (USA)

Conference Chairs

Monte D. Turner, Defense Advanced Research Projects Agency (USA)

Gary W. Kamerman, FastMetrix, Inc. (USA)

Program Committee

Ravil R. Agishev, Kazan State University (Russia)

Phillip Gatt, Lockheed Martin Coherent Technologies (USA)

Jeffrey W. Grantham, Northrop Grumman Corporation (USA)

Clarke E. Harris, FastMetrix, Inc. (USA)

Robert O. Hauge, National Reconnaissance Office (USA)

Richard M. Heinrichs, MIT Lincoln Laboratory (USA)

James C. Lamoreux, NASA Johnson Space Center (USA)

Vasyl Molebny, National Taras Shevchenko University of Kyiv (Ukraine)

William A. Neuman, Lawrence Livermore National Laboratory (USA)

Vladimir L. Pavlovitch, Polyus Research and Development Institute (Russia)

C. Russell Philbrick, The Pennsylvania State University (USA)

Michael W. Roth, Johns Hopkins University (USA)

Jean-Robert Simard, Defence Research and Development Canada (Canada)

Upendra N. Singh, NASA Langley Research Center (USA)

Bevan D. Staple, Ball Aerospace \& Technologies Corporation (USA)

Ove K. Steinvall, Swedish Defence Research Agency (Sweden)

David M. Tratt, The Aerospace Corporation (USA) 
Session Chairs

13 S Imaging Lidar Systems

Monte D. Turner, Defense Advanced Research Projects Agency (USA)

2 Coherent Lidar Applications I

Phillip Gatt, Lockheed Martin Coherent Technologies (USA)

3 Coherent Lidar Applications II

Phillip Gatt, Lockheed Martin Coherent Technologies (USA)

$4 \quad$ Ladar System Modeling and Calibration

Ove K. Steinvall, Swedish Defence Research Agency (Sweden)

5 Emerging Technologies for Lidar

Gary W. Kamerman, FastMetrix, Inc. (USA)

6 Imaging Through Obscurants

Gary W. Kamerman, FastMetrix, Inc. (USA) 